\title{
Corticosteroid-sparing therapy: practice patterns among uveitis specialists
}

\author{
Elizabeth Esterberg • Nisha R. Acharya
}

Received: 8 October 2011 / Accepted: 20 October 2011 /Published online: 6 November 2011

(C) The Author(s) 2011. This article is published with open access at SpringerLink.com

\begin{abstract}
Purpose This study aims to determine uveitis specialists' practice patterns, preferences, and perceptions of corticosteroidsparing therapies for the initial treatment of chronic noninfectious uveitis.

Methods A survey was distributed to the American Uveitis Society and Proctor email listservs in order to restrict the respondents to specialists who likely have extensive experience in the use of immunomodulatory therapy. Topics included effectiveness, usage, and preferences related to seven immunomodulatory treatments.

Results Among the 45 responders, the majority (59\%) had greater than 10 years of experience treating uveitis. Methotrexate was the most commonly used initial therapy for anterior, intermediate, and posterior/panuveitis $(85 \%$, $57 \%$, and $37 \%$ ), and the most preferred for anterior (55\%). Mycophenolate mofetil was the most preferred for intermediate $(35 \%)$ and posterior/panuveitis $(42 \%)$. Primary reasons not to prescribe a treatment were effectiveness for azathioprine, safety/tolerability for cyclosporine and cyclophosphamide, and a mixture of cost, safety/tolerability, and difficulty of administration for the biologic drugs.

Conclusions Within the group of highly experienced uveitis specialists, methotrexate is still the most commonly used initial treatment. Although newer biologic drugs are seen as
\end{abstract}

E. Esterberg $\cdot$ N. R. Acharya $(\square)$

F.I. Proctor Foundation, University of California San Francisco, Room S309, 513 Parnassus Avenue,

San Francisco, CA 94143-0412, USA

e-mail: nisha.acharya@ucsf.edu

N. R. Acharya

Department of Ophthalmology, University of California,

San Francisco,

San Francisco, CA, USA effective, they are not commonly used, or even preferred, as initial corticosteroid-sparing treatment.

Keywords Uveitis · Treatment · Immunomodulatory therapy $\cdot$ Corticosteroids $\cdot$ Biologics $\cdot$ Survey

\section{Introduction}

Uveitis is a set of conditions characterized by intraocular inflammation and is a significant cause of vision loss in the USA and the world [1,2]. Some forms of acute uveitis may be effectively treated with short courses of corticosteroids. Conversely, uveitis that is determined to be chronic and non-infectious in nature often requires the introduction of a corticosteroid-sparing immunomodulatory treatment in order to control inflammation and avoid undesirable complications associated with chronic use of high-dose corticosteroids [3, 4]. Current guidelines recommend starting a corticosteroid-sparing treatment if a dose of greater than $10 \mathrm{mg}$ of oral prednisone is required chronically to control inflammation [3].

A number of immunomodulatory therapy classes are currently used to treat uveitis, including antimetabolites, calcineurin inhibitors, alkylating agents, and biologic drugs. With the exception of the antimetabolites methotrexate and azathioprine, all of these drugs have been introduced in the last 25 years. Due to the low prevalence of uveitis, new treatments have historically been integrated into practice as a result of their success in controlling other autoimmune inflammatory disorders and subsequent anecdotal evidence based on small case series published by uveitis specialists. As collective experience builds, additional evidence has become available in the form of larger retrospective cohort studies and a few small clinical trials. 
Guidelines for the use of corticosteroid-sparing immunomodulatory treatments have been established fairly recently to aid clinicians in treating uveitis, but they do not dictate a specific algorithm on how immunomodulatory therapies should be used [3]. There is little information available on what treatments are being used as first-line corticosteroid-sparing agents and the reasons why specific therapies are not preferred. This survey aims to capture the practice patterns and perceptions of uveitis specialists concerning first-line corticosteroid-sparing treatment for chronic uveitis.

\section{Materials and methods}

\section{Survey population}

The survey was distributed by email to 205 members of the American Uveitis Society and Proctor Foundation listservs through the use of the web application surveymonkey.com. The first email, containing a link to the survey, was sent on 10/ 6/09. All responses were anonymous. The American Uveitis Society is a selective group of uveitis specialists. Admission is voted on by an executive committee and requires applicants to commit at least one third of their time to clinical care and/or research involving immunology/inflammation, at least two first or second author publications on immunology/inflammation in peer-reviewed journals in the last 4 years, and two letters of recommendation, with at least one from a member of the American Uveitis Society. The Proctor Foundation listerv is comprised of specialists in ocular inflammatory disease. There is overlap in these two listservs, but each respondent was only allowed to submit one survey response. This population was chosen despite its small size in order to elicit opinions only from uveitis experts who are likely to have extensive experience with the use of immunomodulatory therapies as steroid-sparing treatment.

\section{Survey}

The survey consisted of five sections and included seven immunomodulatory therapies: methotrexate, mycophenolate mofetil, azathioprine, cyclosporine, cyclophosphamide, infliximab, and adalimumab. The first section contained Likert scales for rating the effectiveness of seven therapies for controlling inflammation and allowing a successful corticosteroid taper in three anatomical locations of uveitis (anterior, intermediate, and posterior/panuveitis). Effectiveness ratings were reported on a four-point Likert scale, with 1 and 2 representing unfavorable responses of "Not effective" and "Somewhat effective", and 3 and 4 representing favorable responses of "Mostly effective" and "Very effective". The second and third sections captured which treatments are most commonly used as first-line corticosteroid-sparing therapy and which would be preferred in an ideal world where cost and availability were not an issue. In these sections, respondents were asked to rankorder the seven treatments within each anatomic location according to actual use and preference. The fourth section had a variety of questions to determine perceived disadvantages of each treatment, as well as more detailed questions on the administration of methotrexate and what dose levels of systemic and topical corticosteroids are considered acceptable maintenance doses. Finally, demographic information on the respondents was collected. Institutional review board exemption was obtained.

\section{Statistical methods}

Descriptive analyses were conducted with binomial 95\% confidence intervals where appropriate. Additional analyses of Likert-style questions were conducted using KruskalWallis analysis of variance to compare effectiveness of treatments [5]. $P$ values shown are nominal (16 hypothesis tests were performed) and only referred to as statistically significant when the reported value was less than 0.003 according to the Bonferroni correction for multiple comparisons. Worth estimates were calculated based on ranking data using a Bradley-Terry model to represent what fraction of overall worth could be assigned to each treatment and to estimate an overall ranking [6]. All analyses were conducted using the statistical software R (www.R-project.org).

\section{Results}

Physician demographics and characteristics

Of the 205 clinicians contacted through the listserv, 45 completed the survey. All respondents characterized themselves as practicing uveitis specialists, of which the majority $(59 \%)$ had more than 10 years of experience, and an additional $14 \%$ had $6-10$ years of experience. Sixty-eight percent of respondents practice in a university or academic setting, $27 \%$ in a private solo/group practice, and $5 \%$ in a health maintenance organization. Ninety-seven percent prescribe and manage immunomodulatory therapy themselves at least some of the time, with $41 \%$ reporting that they always manage such treatment themselves. Eighty-three percent of the respondents practice in the USA, $6 \%$ in Mexico, $6 \%$ in Europe, $3 \%$ in Australia, and 3\% in Canada.

Treatment effectiveness

Treatment effectiveness was defined as the ability to control ocular inflammation and successfully taper corticosteroids to an 
acceptable maintenance dose. According to our respondents, the median acceptable maintenance dose of oral prednisone (i.e., corticosteroid-sparing) was $7.5 \mathrm{mg} /$ day (range 0 to $10 \mathrm{mg} /$ day), and the median acceptable dose of topical prednisolone acetate $1 \%$ was 2 drops/day (range 1 to 6 drops/day).

The median efficacy ratings for each corticosteroid-sparing treatment and anatomic location are shown in Table 1. Within each anatomic location, respondents thought there were significant differences between the effectiveness levels of the seven drugs $(P \leq 0.001)$. As for perceived differences in the effectiveness of each drug within a specific anatomic location, only methotrexate had a statistically significant difference. Respondents considered methotrexate to be only somewhat effective for treating patients with intermediate, posterior, and panuveitis, but mostly effective for those with anterior uveitis $(P \leq 0.001)$. This perceived difference in effectiveness was reflected in the favorability ratings for methotrexate, which were $62 \%$ for anterior, $44 \%$ for intermediate, and $22 \%$ for posterior and panuveitis (Table 2). Adalimumab was considered to be mostly effective for patients with intermediate, posterior, and panuveitis, and very effective for anterior uveitis $(P=0.04)$. Similarly, reported effectiveness ratings for cyclosporine were higher in intermediate and posterior/panuveitis compared to anterior uveitis $(P=0.08)$. Only $20 \%$ responded favorably concerning the use of cyclosporine in patients with anterior uveitis, while $38 \%$ and $44 \%$ assigned a favorable rating for its use in intermediate and posterior/panuveitis, respectively. Infliximab had the highest overall favorability ratings for effectiveness $(82 \%, 69 \%, 71 \%$ for anterior, intermediate, and posterior/panuveitis, respectively) while azathioprine had the lowest $(29 \%, 31 \%, 33 \%)$.

Approximately $60 \%$ of respondents believe that methotrexate is more effective when administered subcutaneously compared to by mouth. However, of patients prescribed methotrexate doses of $20 \mathrm{mg} /$ week and $25 \mathrm{mg} /$ week, only an average of $19 \%$ and $25 \%$, respectively, were placed on subcutaneous treatment. The mean maintenance dose of methotrexate used was $18.5 \mathrm{mg}$ weekly (range 7.0 to $25.0 \mathrm{mg}$ weekly).
Used vs. preferred immunomodulatory therapy

The majority of respondents reported that methotrexate was their most commonly used initial corticosteroid-sparing treatment for noninfectious uveitis in all three anatomic subgroups, followed by mycophenolate mofetil ( $85 \%$ vs. $6 \%$ for anterior, $P<0.001 ; 57 \%$ vs. $22 \%$ for intermediate, $P=$ $0.002 ; 37 \%$ vs. $27 \%$ for posterior/panuveitis, $P=0.49$ ) (see Fig. 1). Azathioprine, cyclophosphamide, and infliximab were rarely or never used as initial corticosteroid-sparing treatment, and none of the respondents listed adalimumab as being used for initial treatment for any anatomic subgroup.

When asked about a scenario where cost and availability of therapies were not an issue, 55\% still reported that methotrexate was their most preferred initial corticosteroidsparing treatment for anterior uveitis, followed by $20 \%$ for mycophenolate mofetil $(P=0.003)$. For intermediate and posterior/panuveitis, however, mycophenolate mofetil was most frequently preferred, followed by methotrexate $(35 \%$ vs. $24 \%$ for intermediate, $P=0.45 ; 42 \%$ vs. $18 \%$ for posterior/panuveitis, $P=0.04)$. Some respondents reported that they would prefer to use infliximab (10\% anterior, $19 \%$ intermediate, $18 \%$ posterior/panuveitis) and adalimumab ( $13 \%$ anterior, $11 \%$ intermediate, $11 \%$ posterior/panuveitis) as initial treatment in each subgroup. Worth estimates based on ranking data indicate that methotrexate and mycophenolate mofetil are consistently ranked first or second overall in all scenarios, while cyclosporine, azathioprine, adalimumab, and infliximab are clustered in the middle and cyclophosphamide is consistently ranked last. The exact order of treatment use and preference fluctuated depending on anatomic location (Table 3).

Reasons immunomodulatory therapies are not prescribed

Respondents indicated that they might choose not to prescribe each of the drugs for different reasons (Fig. 2). The most common reason not to prescribe methotrexate or azathioprine was concern about effectiveness $(42 \%$ and $36 \%$, respectively), although there was also some concern

Table 1 Median effectiveness ratings ${ }^{\mathrm{a}}$ of immunomodulatory treatments

\begin{tabular}{lllllllll}
\hline & MTX & MMF & AZA & CSA & CTX & INF & ADA \\
\hline Anterior & 3 & 3 & 2 & 2 & 4 & 4 & 4 & $<0.001$ \\
Intermediate & 2 & 3 & 2 & 2.5 & 4 & 3 & 3 \\
Posterior/panuveitis & 2 & 3 & 2 & 3 & 4 & 3.5 & 3 & $<0.001$ \\
& $<0.001$ & 0.44 & 0.79 & 0.08 & 0.87 & 0.47 & 0.04 & $P$ value \\
\hline
\end{tabular}

MTX methotrexate, MMF mycophenolate mofetil, AZA azathioprine, CSA cyclosporine, CTX cyclophosphamide, INF infliximab, ADA adalimumab

${ }^{a}$ Respondents were asked to rate the effectiveness of each immunomodulatory therapy by anatomic subtype on a four-point scale $(1=$ not effective, $2=$ somewhat effective, $3=$ mostly effective, $4=$ very effective) 
Table 2 Favorability ratings of immunomodulatory therapies

\begin{tabular}{|c|c|c|c|c|c|c|c|}
\hline & MTX & MMF & AZA & CSA & CTX & $\mathrm{INF}$ & $\mathrm{ADA}$ \\
\hline \multicolumn{8}{|l|}{ Favorable $^{\mathrm{a}}$} \\
\hline Anterior & $28(62 \%)$ & $24(53 \%)$ & $13(29 \%)$ & $9(20 \%)$ & $21(47 \%)$ & $37(82 \%)$ & $33(73 \%)$ \\
\hline Intermediate & $20(44 \%)$ & $27(60 \%)$ & $14(31 \%)$ & $17(38 \%)$ & $24(53 \%)$ & $31(69 \%)$ & $26(58 \%)$ \\
\hline Posterior/panuveitis & $10(22 \%)$ & $24(53 \%)$ & $15(33 \%)$ & $20(44 \%)$ & $32(71 \%)$ & $32(71 \%)$ & $25(56 \%)$ \\
\hline \multicolumn{8}{|l|}{ No opinion } \\
\hline Anterior & $0(0 \%)$ & $7(16 \%)$ & $19(42 \%)$ & $16(36 \%)$ & $20(44 \%)$ & $5(11 \%)$ & $10(22 \%)$ \\
\hline Intermediate & $1(2 \%)$ & $4(9 \%)$ & $13(29 \%)$ & $11(24 \%)$ & $17(38 \%)$ & $7(16 \%)$ & $12(27 \%)$ \\
\hline Posterior/panuveitis & $1(2 \%)$ & $2(4 \%)$ & $11(24 \%)$ & $7(16 \%)$ & $9(20 \%)$ & $7(16 \%)$ & $16(36 \%)$ \\
\hline
\end{tabular}

MTX methotrexate, MMF mycophenolate mofetil, AZA azathioprine, CSA cyclosporine, CTX cyclophosphamide, INF infliximab, ADA adalimumab

"Rating question responses of "mostly effective" or "very effective" were considered favorable. Respondents could also indicate if they had no opinion for a particular combination

about safety/tolerability ( $13 \%$ and $18 \%)$. Common reasons not to prescribe mycophenolate mofetil included cost $(40 \%)$, lack of long-term data on its use $(13 \%)$, and safety/tolerability $(13 \%)$. The primary concern with using cyclosporine or cyclophosphamide was safety/tolerability (44\% and $80 \%$ ), but other reasons not to prescribe cyclosporine included effectiveness (38\%) and cost (13\%). The most common reason not to prescribe infliximab and adalimumab was cost (62\% and $56 \%$, respectively). Other prominent concerns for these biologic drugs included a lack of long-term data (24\% and $29 \%)$, safety/tolerability $(22 \%$ and $20 \%$ ), and difficulty of administration (38\% and 13\%).

\section{Discussion}

Our results indicate that within each anatomic location, there are significant differences in the perceived effective- ness of corticosteroid-sparing treatments for controlling ocular inflammation and allowing a successful corticosteroid taper.

Though they do not receive the highest favorability ratings for effectiveness, the most commonly used and most preferred drugs are from the antimetabolite class. Respondents indicate that the long-used methotrexate is their most common first choice corticosteroid-sparing therapy for all anatomic locations of uveitis, with only mild concerns for safety and tolerability. Even given the choice of any other drug disregarding cost and availability, most would still prefer to prescribe methotrexate for anterior uveitis. Methotrexate, however, was the only drug in which there was a significant difference in effectiveness ratings by anatomic location. For intermediate and posterior/panuveitis, respondents would prefer to use mycophenolate mofetil; the main reason for not prescribing mycophenolate initially seems to be cost. These trends were seen when looking at
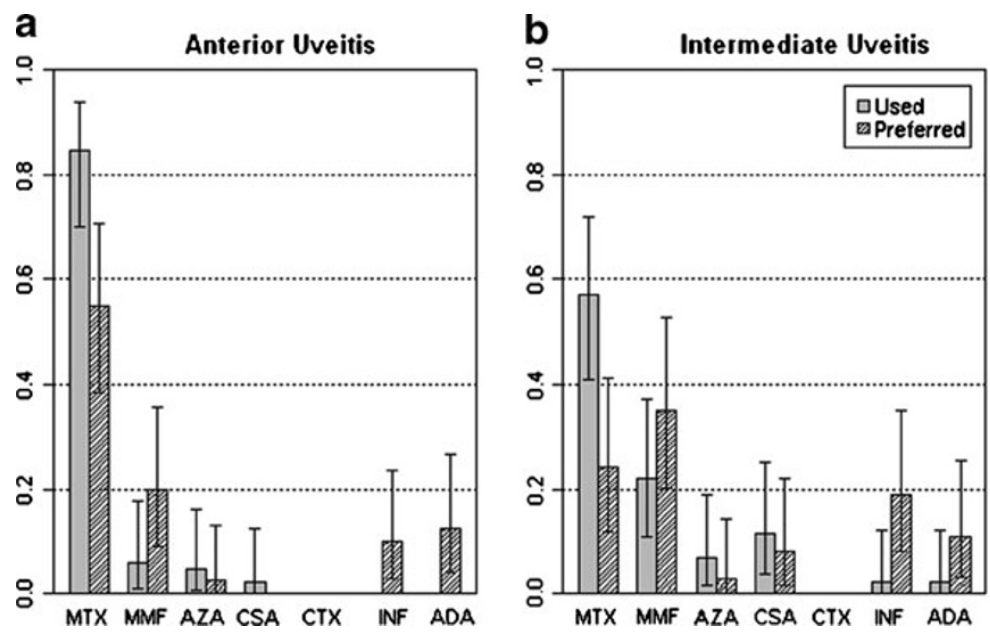

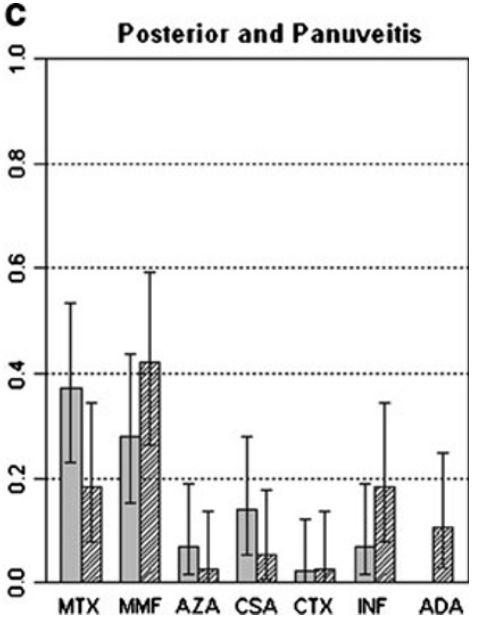

Fig. 1 Histograms showing proportions of respondents, with $95 \%$ onfidence intervals, ranking each treatment as most commonly used or most preferred if cost and availability were not an issue. MTX methotrexate,
$M M F$ mycophenolate mofetil, $A Z A$ azathioprine, $C S A$ cyclosporine, $C T X$ cyclophosphamide, $I N F$ infliximab, $A D A$ adalimumab 
Table 3 Overall rankings by anatomic location of uveitis and corresponding worth estimates ${ }^{\mathrm{a}}$

\begin{tabular}{|c|c|c|c|c|c|c|}
\hline \multirow[t]{2}{*}{ Rank } & \multicolumn{2}{|l|}{ Anterior } & \multicolumn{2}{|l|}{ Intermediate } & \multicolumn{2}{|l|}{ Posterior/pan } \\
\hline & Used & Preferred & Used & Preferred & Used & Preferred \\
\hline 1 & MTX (0.60) & MTX (0.30) & MTX (0.24) & MMF (0.23) & MMF (0.21) & MMF (0.22) \\
\hline 2 & MMF (0.13) & MMF (0.22) & $\operatorname{MMF}(0.21)$ & $\operatorname{MTX}(0.20)$ & MTX (0.19) & MTX (0.18) \\
\hline 3 & INF $(0.07)$ & INF $(0.14)$ & AZA (0.13) & INF $(0.16)$ & AZA (0.14) & INF $(0.15)$ \\
\hline 4 & AZA (0.07) & $\operatorname{ADA}(0.13)$ & CSA $(0.13)$ & $\mathrm{ADA}(0.13)$ & CSA $(0.14)$ & $\operatorname{ADA}(0.15)$ \\
\hline 5 & ADA (0.06) & AZA $(0.10)$ & INF $(0.13)$ & AZA (0.12) & INF (0.13) & CSA $(0.12)$ \\
\hline 6 & CSA $(0.05)$ & CSA $(0.07)$ & ADA $(0.11)$ & CSA $(0.11)$ & ADA (0.11) & AZA (0.11) \\
\hline 7 & CTX (0.02) & CTX (0.04) & CTX (0.05) & CTX (0.05) & CTX (0.08) & CTX (0.07) \\
\hline
\end{tabular}

$M T X$ methotrexate, $M M F$ mycophenolate mofetil, $A Z A$ azathioprine, $C S A$ cyclosporine, $C T X$ cyclophosphamide, INF infliximab, $A D A$ adalimumab

${ }^{\text {a }}$ Calculated using a Bradley-Terry model: represent what fraction of overall worth could be assigned to each treatment and are used to estimate an overall ranking (e.g., based on the collective rankings of the respondents, methotrexate could be assigned $60 \%$ of the collective preference as the most used initial treatment for anterior uveitis)

first choices for most commonly used and most preferred treatments and when evaluating the full ranking data using a Bradley-Terry model. This is a well-developed method of rank-ordering items based on how each respondent ranks each item in relation to the others, and may provide a more relevant estimate of the relative usage and preference within our survey population by incorporating more in-depth information [6].

Even with the recent availability of generic mycophenolate mofetil, the cost per month is still more than double that of methotrexate. According to Medicare reimbursement rates, a 1-month supply of maintenance dose methotrexate (25 mg per week) costs $\$ 50.55$, and an equivalent supply of generic mycophenolate mofetil ( $1 \mathrm{~g}$ twice a day) costs
$\$ 118.50$ [7]. Retrospective studies by the Systemic Immunosuppressive Therapy for Eye Diseases Cohort Study Research Group, the largest such studies to date, have reported corticosteroid-sparing success rates at 6 months with methotrexate and mycophenolate for posterior/panuveitis at $21 \%$ and $41 \%$, respectively $[8,9]$. Another study, comprised mostly of patients with posterior/panuveitis, reported success rates of $42 \%$ with methotrexate compared to $79 \%$ with mycophenolate mofetil [10]. There have been no controlled trials comparing methotrexate and mycophenolate mofetil for any anatomic location to confirm the differences found in retrospective studies. Azathioprine, the third antimetabolite option, was the least popular of the three among our respondents. Published retrospective

Fig. 2 Histogram annotated with percentage of respondents, and $95 \%$ confidence intervals, citing each reason for not prescribing specific immunomodulatory therapies. $M T X$ methotrexate, $M M F$ mycophenolate mofetil, $A Z A$ azathioprine, CSA cyclosporine, $C T X$ cyclophosphamide, $I N F$ infliximab, $A D A$ adalimumab

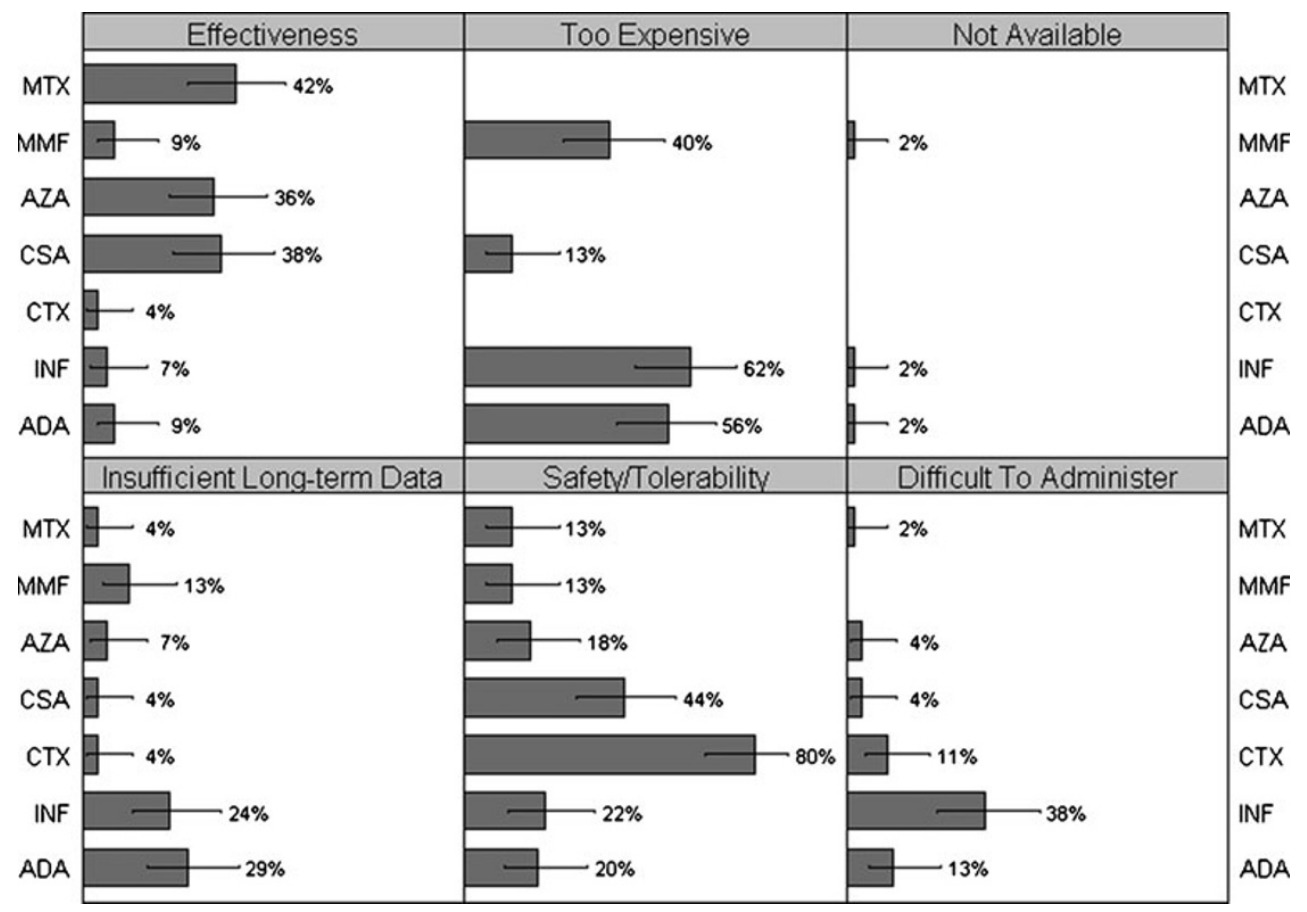


studies report similar effectiveness rates to those of methotrexate for uveitis, but discontinuations due to safety and tolerability may be more frequent [9-12]. Azathioprine is also widely used in rheumatologic diseases and organ transplantation. Randomized trials comparing methotrexate to azathioprine have shown similar effectiveness for treating ANCA-associated vasculitis [13] and myasthenia gravis [14], and mixed results in rheumatoid arthritis with one small trial showing similar effectiveness [15] and another showing greater effectiveness with methotrexate [16]. Mycophenolate mofetil was shown to be more effective than azathioprine in a randomized trial of patients with Crohn's disease [17] and for cardiac transplantation [18], but trials in lupus nephritis [19] and renal transplantation [20] found no significant differences.

Despite being the least used and least preferred as firstline corticosteroid-sparing treatment, the biologic drugs adalimumab and infliximab and the alkylating agent cyclophosphamide received the overall highest effectiveness ratings based on both medians and percent of respondents giving a favorable response. Actual use and preference, however, do not match these reported beliefs. In fact, adalimumab, infliximab, and cyclophosphamide are infrequently used and preferred as initial corticosteroidsparing treatment, even if cost and availability are not an issue. In the case of adalimumab and infliximab, respondents cited a number of reasons not to prescribe these treatments, including cost, insufficient long-term data, concerns with safety and tolerability, and difficulty of administration. Adalimumab is given by subcutaneous injections and infliximab by intravenous infusions. Despite the overwhelming opinion that cyclophosphamide is effective, it was the least preferred drug, primarily due to concern for the safety of the patient. Cyclophosphamide has been associated with an increased risk of malignancy, infertility, and other undesirable side effects, so this finding was not surprising [21]. Cyclosporine, a calcineurin inhibitor, was not commonly used and rarely preferred as first-line corticosteroid-sparing therapy because of safety and tolerability concerns and some doubt as to its effectiveness. Previous publications report widely varying rates of success with cyclosporine for uveitis, and there is evidence of high rates of side effects including nephrotoxicity [22].

This study does have limitations. It is possible that those who chose to participate in the survey are somehow different from those who did not, which would affect the generalizability of our results to all uveitis specialists or even to the members of the listservs as a whole. The sample size raises the question of whether the responses collected in this study reflect actual practice patterns of uveitis specialists, though studies have shown surveys with similar response rates (about 25\%) to have comparable results to those with higher response rates [23-25]. Increased sample size could have been achieved by including a broader sample of ophthalmologists in the study, but this would have potentially compromised our goal of eliciting opinions from uveitis specialists with extensive experience with immunomodulatory therapy. The respondents were all uveitis specialists, and the majority had greater than 10 years of practice experience, likely indicating the respondent group had a high level of expertise in this subject area. This is also highlighted by the fact that $100 \%$ of respondents reported maximum oral corticosteroid doses of $10 \mathrm{mg}$ /day or less as acceptable maintenance levels, demonstrating familiarity with the SUN guidelines on the use of immunosuppressives [3]. This is in contrast with the population of a recent survey focusing on corticosteroid use in which steroid-sparing immunosuppressives were rarely used and an average prednisone maintenance dose of $34 \mathrm{mg} /$ day was reported [26]. In addition, as the vast majority of the respondents in our survey practice in the USA, reported practice patterns and preferences may be specific to the USA and could be different in other countries.

Perceptions of effectiveness and negative aspects of each treatment could be affected in a number of ways. Although general guidelines for the use of immunomodulatory treatment of noninfectious uveitis have been established, uveitis specialists may differ in the way they use each treatment (varying maintenance doses, etc). They may also treat patients with varying disease severities and etiologies of uveitis, which could affect perceptions about each treatment. It is also important to note that this survey reported usage and preferences based on anatomical location of inflammation rather than any associated disease entity. For patients with severe uveitis-related complications or a known associated inflammatory disease such as juvenile idiopathic arthritis or Behcet's, the nature of the results may have been different. Other factors such as comanagement with a rheumatologist and age of the patient population may also affect practice patterns.

Additionally, not all immunomodulatory drugs currently used to treat uveitis were included in the survey; omitted treatments include the calcineurin inhibitors tacrolimus and sirolimus, the alkylating agent chlorambucil, and newer biologic agents such as golimumab or certolizumab. Etanercept was also not included; though etanercept has been commercially available since 1999 and is used to treat various systemic inflammatory conditions, a number of studies have indicated that this particular TNF-alpha inhibitor is likely not effective for controlling ocular inflammation [27-29]. There was initially some concern in the rheumatology literature that etanercept may even induce ocular inflammation, but more recent findings support the continued use of etanercept therapy for 
inflammatory diseases with the caveat that patients developing uveitis may require a change in treatment regimen [30]. We chose to include treatments which have been most commonly reported in the literature to ensure that most respondents would have some experience with each, making comparisons between them possible.

Despite potentially mitigating factors, the results of this survey were striking. They raise questions that warrant further study, including the possibility of variable effectiveness by anatomic location for some treatments, and also highlight factors that uveitis specialists feel limit the practical use of each immunomodulatory treatment. These results may help guide future research comparing treatment effectiveness for initial corticosteroid-sparing therapy in noninfectious uveitis.

Acknowledgments We thank the American Uveitis Society for granting permission to send the survey to their email listserv.

Declaration of interest None of the authors have any conflicts of interest related to any of the content in this paper. The authors alone are responsible for the content and writing of the paper.

Research funding Nisha R. Acharya, MD, MS is supported by a National Eye Institute grant K23EY017897 and a Research to Prevent Blindness Career Development Award. This work was also supported by a NEI EY02162 grant to the Department of Ophthalmology and an unrestricted grant from the Research to Prevent Blindness Foundation.

Open Access This article is distributed under the terms of the Creative Commons Attribution License which permits any use, distribution and reproduction in any medium, provided the original author(s) and source are credited.

\section{References}

1. Nussenblatt RB (1990) The natural history of uveitis. Int Ophthalmol 14(5-6):303-308

2. Rothova A, Suttorp-van Schulten MS, Frits Treffers W, Kijlstra A (1996) Causes and frequency of blindness in patients with intraocular inflammatory disease. Br J Ophthalmol 80(4):332-336

3. Jabs DA, Rosenbaum JT, Foster CS, Holland GN, Jaffe GJ, Louie JS, Nussenblatt RB, Stiehm ER, Tessler H, Van Gelder RN, Whitcup SM, Yocum D (2000) Guidelines for the use of immunosuppressive drugs in patients with ocular inflammatory disorders: recommendations of an expert panel. Am J Ophthalmol 130(4):492-513

4. Stanbury RM, Graham EM (1998) Systemic corticosteroid therapy - side effects and their management. $\mathrm{Br} \mathrm{J}$ Ophthalmol 82(6):704-708

5. Kruskal WH, Wallis WA (1952) Use of ranks in one-criterion variance analysis. Am Stat Assoc 47:583-621

6. Bradley RA, Terry ME (1952) Rank analysis of incomplete block designs I: the method of paired comparisons. Biometrika 39:324345

7. Drug Topics Red Book (2009). Thomson Healthcare, Montvale, NJ. 113(319):574-575
8. Daniel E, Thorne JE, Newcomb CW, Pujari SS, Kacmaz RO, Levy-Clarke GA, Nussenblatt RB, Rosenbaum JT, Suhler EB, Foster CS, Jabs DA, Kempen JH (2010) Mycophenolate mofetil for ocular inflammation. Am J Ophthalmol 149(3):423432, e1-e2

9. Gangaputra S, Newcomb CW, Liesegang TL, Kacmaz RO, Jabs DA, Levy-Clarke GA, Nussenblatt RB, Rosenbaum JT, Suhler EB, Thorne JE, Foster CS, Kempen JH (2009) Methotrexate for ocular inflammatory diseases. Ophthalmology 116(11):21882198, e2181

10. Galor A, Jabs DA, Leder HA, Kedhar SR, Dunn JP, Peters GB 3rd, Thorne JE (2008) Comparison of antimetabolite drugs as corticosteroid-sparing therapy for noninfectious ocular inflammation. Ophthalmology 115(10):1826-1832

11. Andrasch RH, Pirofsky B, Burns RP (1978) Immunosuppressive therapy for severe chronic uveitis. Arch Ophthalmol 96(2):247251

12. Pasadhika S, Kempen JH, Newcomb CW, Liesegang TL, Pujari SS, Rosenbaum JT, Thorne JE, Foster CS, Jabs DA, Levy-Clarke GA, Nussenblatt RB, Suhler EB (2009) Azathioprine for ocular inflammatory diseases. Am J Ophthalmol 148(4):500-509, e502

13. Pagnoux C, Mahr A, Hamidou MA, Boffa JJ, Ruivard M, Ducroix JP, Kyndt X, Lifermann F, Papo T, Lambert M, Le Noach J, Khellaf M, Merrien D, Puechal X, Vinzio S, Cohen P, Mouthon L, Cordier JF, Guillevin L (2008) Azathioprine or methotrexate maintenance for ANCA-associated vasculitis. N Engl J Med 359 (26):2790-2803

14. Heckmann JM, Rawoot A, Bateman K, Renison R, Badri M (2011) A single-blinded trial of methotrexate versus azathioprine as steroid-sparing agents in generalized myasthenia gravis. BMC Neurol 11:97

15. Hamdy H, McKendry RJ, Mierins E, Liver JA (1987) Low-dose methotrexate compared with azathioprine in the treatment of rheumatoid arthritis. A twenty-four-week controlled clinical trial. Arthritis Rheum 30(4):361-368

16. Jeurissen ME, Boerbooms AM, van de Putte LB, Doesburg WH, Mulder J, Rasker JJ, Kruijsen MW, Haverman JF, van Beusekom HJ, Muller WH et al (1991) Methotrexate versus azathioprine in the treatment of rheumatoid arthritis. A forty-eight-week randomized, double-blind trial. Arthritis Rheum 34(8):961-972

17. Neurath MF, Wanitschke R, Peters M, Krummenauer F, Buschenfelde KH Meyer zum, Schlaak JF (1999) Randomised trial of mycophenolate mofetil versus azathioprine for treatment of chronic active Crohn's disease. Gut 44(5):625-628

18. Eisen HJ, Kobashigawa J, Keogh A, Bourge R, Renlund D, Mentzer R, Alderman E, Valantine H, Dureau G, Mancini D, Mamelok R, Gordon R, Wang W, Mehra M, Constanzo MR, Hummel M, Johnson J (2005) Three-year results of a randomized, double-blind, controlled trial of mycophenolate mofetil versus azathioprine in cardiac transplant recipients. J Heart Lung Transplant 24(5):517-525

19. Houssiau FA, D'Cruz D, Sangle S, Remy P, Vasconcelos C, Petrovic R, Fiehn C, de Ramon GE, Gilboe IM, Tektonidou M, Blockmans D, Ravelingien I, le Guern V, Depresseux G, Guillevin L, Cervera R (2010) Azathioprine versus mycophenolate mofetil for long-term immunosuppression in lupus nephritis: results from the MAINTAIN Nephritis Trial. Ann Rheum Dis 69(12):20832089

20. Remuzzi G, Lesti M, Gotti E, Ganeva M, Dimitrov BD, EneIordache B, Gherardi G, Donati D, Salvadori M, Sandrini S, Valente U, Segoloni G, Mourad G, Federico S, Rigotti P, Sparacino V, Bosmans JL, Perico N, Ruggenenti P (2004) Mycophenolate mofetil versus azathioprine for prevention of acute rejection in renal transplantation (MYSS): a randomised trial. Lancet 364(9433):503-512 
21. Kempen JH, Gangaputra S, Daniel E, Levy-Clarke GA, Nussenblatt RB, Rosenbaum JT, Suhler EB, Thorne JE, Foster CS, Jabs DA, Helzlsouer KJ (2008) Long-term risk of malignancy among patients treated with immunosuppressive agents for ocular inflammation: a critical assessment of the evidence. Am J Ophthalmol 146(6):802812, e801

22. Kacmaz RO, Kempen JH, Newcomb C, Daniel E, Gangaputra S, Nussenblatt RB, Rosenbaum JT, Suhler EB, Thorne JE, Jabs DA, Levy-Clarke GA, Foster CS (2010) Cyclosporine for ocular inflammatory diseases. Ophthalmology 117(3):576-584

23. Visser PS, Krosnick JA, Marquette J, Curtin M (1996) Mail surveys for election forecasting? An evaluation of the Columbus Dispatch poll. Public Opin Q 60(2):181-227

24. Keeter S, Kennedy C, Dimock M, Best J, Craighill P (2006) Gauging the impact of growing nonresponse on estimates from a national RDD telephone survey. Public Opin Q 70(5):759-779

25. Curtin R, Presser S, Singer E (2000) The effects of response rate changes on the index of consumer sentiment. Public Opin Q 64 (4):413-428
26. Nguyen QD, Hatef E, Kayen B, Macahilig CP, Ibrahim M, Wang J, Shaikh O, Bodaghi B (2011) A cross-sectional study of the current treatment patterns in noninfectious uveitis among specialists in the United States. Ophthalmology 118(1):184-190

27. Foster CS, Tufail F, Waheed NK, Chu D, Miserocchi E, Baltatzis S, Vredeveld CM (2003) Efficacy of etanercept in preventing relapse of uveitis controlled by methotrexate. Arch Ophthalmol 121(4):437-440

28. Galor A, Perez VL, Hammel JP, Lowder CY (2006) Differential effectiveness of etanercept and infliximab in the treatment of ocular inflammation. Ophthalmology 113(12):2317-2323

29. Smith JR, Levinson RD, Holland GN, Jabs DA, Robinson MR, Whitcup SM, Rosenbaum JT (2001) Differential efficacy of tumor necrosis factor inhibition in the management of inflammatory eye disease and associated rheumatic disease. Arthritis Rheum 45 (3):252-257

30. Lim LL, Fraunfelder FW, Rosenbaum JT (2007) Do tumor necrosis factor inhibitors cause uveitis? A registry-based study. Arthritis Rheum 56(10):3248-3252 